\title{
Caracterização de compósitos de alumínio reforçado com partículas quasicristalinas produzidos por stir casting/laminação
}

\author{
Characterization of reinforced aluminum \\ composites with quasicrystalline particles \\ produced by stir casting/rolling
}

\author{
Maria Aline Martins Gonzaga ${ }^{1}$, Tibério Andrade dos Passos ${ }^{2}$, \\ Rodinei Medeiros Gomes ${ }^{3}$
}

\footnotetext{
${ }^{1,2}$ Laboratório de Solidificação Rápida - LSR - Departamento de Engenharia de Materiais/CT/UFPB, CEP: 58051-900, João Pessoa, PB, Brasil.

e-mail: mr.alinemartins@gmail.com, professor.tiberio@gmail.com

${ }^{3}$ Laboratório de Solidificação Rápida - LSR - Departamento de Engenharia Mecânica/CT/UFPB, CEP: 58051-900, João

Pessoa, PB, Brasil.

e-mail: rodineix@gmail.com
}

\begin{abstract}
RESUMO
A exigência contínua de materiais leves e de alto desempenho para as indústrias aeroespaciais e automotivas, levou ao desenvolvimento de materiais compósitos com matriz metálica, destacando-se entre eles, àqueles de base alumínio. Os quasicristais são materiais metálicos com uma estrutura atômica complexa, a qual resulta uma combinação de propriedades físico-mecânicas similares às de algumas cerâmicas frágeis e compostos intermetálicos, como elevada resistência e dureza, elevado módulo de elasticidade, baixo coeficiente de atrito e boa resistência ao desgaste e à corrosão. Essas propriedades são bastante promissoras para aplicação industrial, mas a fragilidade dos quasicristais restringe sua aplicação como fase de reforço em compósitos, podendo substituir os reforços cerâmicos utilizados tradicionalmente, como $\mathrm{SiC}$ e $\mathrm{Al}_{2} \mathrm{O}_{3}$. No presente trabalho, compósitos de matriz de alumínio reforçado com 2,5\%, 5\% e 10\% em volume de partículas quasicristalinas, foram produzidas através do método de agitação do banho de metal fundido stir casting. Etapas subsequentes de conformação por laminação foram realizadas em todos os compósitos alumínio/quasicrsital. A liga quasicristalina $\mathrm{Al}_{61,7} \mathrm{Cu}_{25,5} \mathrm{Fe}_{12,3} \mathrm{Mn}_{0,5}$ foi obtida via fusão convencional e posterior tratamento térmico. A moagem da liga quasicristalina, para obtenção de pós, foi realizada em moinho de alta energia. A microestrutura dos quasicristais foi investigada através da Difração de Raios-X e da Microscopia Eletrônica de Varredura (MEV). A distribuição das partículas quasicristalinas na matriz de alumínio e a influência na dureza dos compósitos foram investigadas por MEV e Microdureza Vickers, respectivamente. Os compósitos apresentaram uma boa distribuição das partículas quasicristalinas na matriz de alumínio, embora ocorrido em algumas regiões a formação de poros e aglomerados. O processo de laminação promoveu um aumento significativo na dureza em relação aos compósitos obtidos diretamente da fundição.
\end{abstract}

Palavras-chave: alumínio, quasicristal, compósito, laminação.

\section{ABSTRACT}

The continuous requirement of lightweight and high performance materials for the aerospace and automotive industries led to the development of composite materials with a metallic matrix, among which aluminum-based composites. The quasicrystals are metallic materials with a complex atomic structure, which results in a combination of physico-mechanical properties similar to those of some fragile ceramics and intermetallic compounds, such as high strength and hardness, high modulus of elasticity, low coefficient of friction and good resistance to wear and corrosion. These properties are very promising for industrial application, but the fragility of the quasicrystals restricts their application as a reinforcement phase in composites, and can replace the traditionally used ceramic reinforcements such as $\mathrm{SiC}$ and $\mathrm{Al}_{2} \mathrm{O}_{3}$. In the present work, reinforced aluminum matrix composites with $2,5 \%, 5 \%$ and $10 \%$ volume of quasicrystalline particles were produced by the stir cast method of stir casting. Subsequent steps of laminating were performed on all alumi- 
num / quasicrystals composites. The quasicrystalline $\mathrm{Al}_{61,7} \mathrm{Cu}_{25,5} \mathrm{Fe}_{12,3} \mathrm{Mn}_{0,5}$ alloy was obtained by conventional melting and subsequent heat treatment. The mill of the quasicrystalline alloy, to obtain powders, was carried out in a high-energy mill. The microstructure of quasicrystals was investigated by X-ray Diffraction and Scanning Electron Microscopy (SEM). The distribution of the quasicrystalline particles in the aluminum matrix and the influence on the hardness of the composites were investigated by SEM and Vickers Microhardness, respectively. The composites presented a good distribution of the quasicrystalline particles in the aluminum matrix, although in some regions the formation of pores and agglomerates occurred. The rolling process promoted a significant increase in hardness in relation to the composites obtained directly from the casting.

Keywords: aluminum, quasicrystal, composite, rolling.

\section{INTRODUÇÃO}

A necessidade de materiais leves e de alto desempenho levou ao desenvolvimento de materiais Compósitos com Matriz Metálica (CMM). Os CMM geralmente consistem em uma matriz leve reforçada por partículas ou fibras curtas de um material duro. A maioria dos CMM tradicionalmente desenvolvidos são compostos de uma matriz de liga de $\mathrm{Al}$ e/ou $\mathrm{Mg}$ com fases de reforço de material cerâmico frágil, como $\mathrm{SiC}$ e/ou $\mathrm{Al}_{2} \mathrm{O}_{3}$. O uso de partículas quasicristalinas de $\mathrm{AlCuFe}$ em compósitos de matriz metálica consistem em um desafio promissor pra o desenvolvimento de compósitos com propriedades inovadoras e comparáveis ou até melhores que os convencionais com reforço cerâmico já existentes. Em particular, por causa da natureza metálica do quasicristal $\mathrm{AlCuFe}$, melhor compatibilidade na interface matriz/reforço são esperados em comparação com os compósitos de $\mathrm{Al} /$ reforço cerâmico [1,2]. Além disso, atualmente existem uma variedade de trabalhos disponibilizados na literatura, [3,4,5,6], confirmando a importância desse material para aplicação em compósitos com matriz metálica, incluindo aqueles com matriz de alumínio.

Há basicamente dois métodos de processamento comumente utilizados para produzir CMM de base alumínio reforçado com partículas: técnicas de processamento por fase líquida e por fase sólida [7]. Atualmente, as aplicações de CMM estão aumentando progressivamente. Portanto, o foco dos estudos recentes estão no desenvolvimento de novas técnicas de processamento ou na melhoria das técnicas já existentes [8].

O stir casting ou agitação do banho de metal fundido, é um técnica de processamento por fase líquida, a qual consiste na adição de partículas sólidas dentro do banho de metal fundido por meio de pressão ou por deposição promovendo a agitação do banho[9]. Essa técnica de metalurgia líquida é a mais econômica de todas as rotas disponíveis para produção de compósitos com matriz metálica, e permite que componentes de grandes dimensões sejam fabricados. Alguns fatores que devem ser considerados nessa técnica são: a dificuldade de obter uma distribuição uniforme do material de reforço, molhabilidade entre a matriz e o reforço, porosidade no produto final, reação química entre o material da liga matriz e do reforço $[8,10]$.

Etapas subsequentes de processamento podem ser realizadas para adquirir a forma definitiva aos compósitos obtidos pelo processo de fundição, como o stir casting. Uma operação conhecida na conformação mecânica é a laminação, que consiste na passagem de uma peça entre dois cilindros que giram, de forma a reduzir a área da sua secção transversal produzindo placas e chapas. Além da mudança de dimensões, outro resultado obtido comumente através da conformação mecânica é alteração das propriedades do metal, em relação àquelas anteriores ao processamento [11]. Adicionalmente, a etapa de conformação por laminação pode ajudar a obtenção de um produto final com uma estrutura homogênea através da remoção de vazios e micro-vazios, e também contribuir para uma redistribuição das partículas de reforço, resultando em considerável eliminação de aglomerados de partículas, e consequentemente, colaborar para o aumento da resistência mecânica do material. Chapas de ligas de alumínio, obtidas por laminação, são amplamente utilizadas nas indústrias de automóvel, construção naval e aeroespacial, como substitutos de chapas de aço e polímeros reforçados com fibra de vidro, devido às suas excelentes propriedades, tais como boa resistência mecânica, resistência à corrosão, baixo peso e habilidades de solda [12]. A produção de chapas ou placas laminadas de compósitos de alumínio com partículas quasicristalinas pode facilitar a substituição de outros materiais para aplicações que necessitem de uma alta relação resistência/peso.

Não foram encontrados na literatura trabalhos relativos à produção de CMM com adição de partículas quasicristalinas através do método stir casting com posterior processo de conformação por laminação. Porém, o processamento de CMM Alumínio/Quasicristal por meio da união desses dois métodos convencionais mostrou-se ser um processo de fácil operação, com utilização de equipamentos simples e de baixo custo, além da obtenção da propriedade de dureza nos compósitos obtidos, comparável com CMM da mesma natureza obtidos por outros métodos de fabricação [3, 4, 6].

A utilização de conformação por laminação após a obtenção dos CMM de matriz de alumínio com re- 
forço quasicristalino por stir casting foi motivada pelo processo Accumulative Roll Bonding (ARB). O ARB é um processo de deformação plástica severa e foi inicialmente desenvolvido e aplicado a uma pilha de chapas metálicas, com o objetivo de aumentar a resistência mecânica do produto final. Após uma variedade de estudos, esse processo foi aplicado no processamento de CMM reforçado com $\mathrm{SiC}$, utilizando o reforço em pó intercalado entre as chapas metálicas nas etapas de laminação. Como resultado, foram obtidos compósitos com grãos menores e com maior resistência mecânica em ralação a resistência inicial da matriz, fato atríbuido às partículas de reforço. Essas partículas seguram parte da deformação da matriz durante a laminação, aumentando a tensão no material, e consequentemente a resistência mecânica é melhorada [13]. Essa técnica trouxe motivação para utilização da laminação com processo adicional ao stir casting, a fim da obtenção de compósitos com melhores valores de resistência mecânica, devido ao processo de deformação da matriz.

Os quasicristais devido a sua estrutura atômica complexa, possuem uma combinação de propriedades físico-mecânicas únicas, como elevada resistência e dureza, elevado módulo de elasticidade, baixo coeficiente de atrito, baixa energia superficial e boa resistência ao desgaste e à corrosão. As propriedades mecânicas dos materiais quasicristalinos são similares às de algumas cerâmicas frágeis ou compostos intermetálicos [14, 15]. A dureza da liga quasicristalina do sistema $\mathrm{Al}-\mathrm{Cu}-\mathrm{Fe}$ é cerca de $10 \%$ do módulo de Young, a qual está próxima da relação obtida para os diamantes e algumas cerâmicas. Adicionalmente, o aumento da temperatura resulta em significativa diminuição na dureza do quasicristal [3].

Essas propriedades são bastante promissoras para aplicação industrial, mas a fragilidade dos quasicritais restringe sua aplicação como fase de reforço em materiais compósitos e sob forma de revestimento. A pesquisa relacionada aos quasicristais atualmente concentra-se em encontrar técnicas de produção práticas e aplicações para esses materiais [16]. A formação de quasicristais estáveis pode normalmente ser baseada pelos diagramas de fases de equilíbrio. Dessa forma, eles podem ser produzidos por processos de equilíbrio convencionais utilizando procedimentos de fusão e solidificação [17]. Atualmente, Existem cerca de 100 sistemas de ligas metálicas capazes de formarem fases quasicristalinas, ligas de base de alumínio, cobre, galium, magnésio, níquel, titânio, zinco, zircônio, entre outras, são exemplos desses sistemas [18].

Quasicristais estáveis do sistema $\mathrm{Al}-\mathrm{Cu}$-Fe são mais atraentes para utilização em compósitos de base alumínio em comparação aos outros sistemas por causa do baixo custo, fácil acesso, possibilidade de reciclagem dos componentes, ausência de toxicidade, densidade relativamente baixa, alta estabilidade térmica e compatibilidade com matriz de alumínio [19]. A preparação de uma única fase quasicristalina icosaédrica de $\mathrm{Al}-\mathrm{Cu}-\mathrm{Fe}$ por fundição convencional é difícil, devido a sua estreita faixa de composição e, portanto, solidificação rápida e/ou tratamentos térmicos são realizados para evitar segregação [6].

O objetivo deste trabalho é caracterizar microestruturalmente e analisar a evolução da dureza de compósitos da liga quasicristalina do sistema $\mathrm{AlCuFeMn} \mathrm{em} \mathrm{uma} \mathrm{matriz} \mathrm{de} \mathrm{alumínio} \mathrm{reciclado} \mathrm{produzidos} \mathrm{por}$ stir casting/laminação.

\section{MATERIAIS E MÉTODOS}

Todos os procedimentos experimentais foram realizados no Laboratório de Solidificação Rápida (LSR) da Universidade Federal da Paraíba (UFPB).

\subsection{Liga quasicristalina AICuFeMn}

A liga quasicristalina $\mathrm{Al}_{61,7} \mathrm{Cu}_{25,5} \mathrm{Fe}_{12,3} \mathrm{Mn}_{0,5}$ foi produzida por fundição convencional, em um forno de indução equipado com cadinho de soleira fria, sob atmosfera de argônio. Essas ligas foram submetidas ao tratamento térmico, em forno radiante de marca Analogia, modelo NA 800, mantendo a amostra por $12 \mathrm{~h}$ a temperatura de $800^{\circ} \mathrm{C}$. Para a obtenção dos compósitos, fez-se necessário a cominuição da liga quasicristalina, a fim de adicioná-las na forma de pó na matriz de alumínio.

A moagem foi realizada em moinho planetário do tipo Fritsch Pulverisette P5, utilizando uma razão bola/massa de 10/1, com uma rotação de $200 \mathrm{rpm}$ por 1,5 h. As jarras e a bolas utilizadas foram de aço inoxidável.

A liga quasicristalina após tratamento térmico, foi analisada por difração de raios-X. Foi utilizado o difratômetro de Raios-X da marca Siemens, modelo D500, sendo empregada a radiação CuKa, cujo comprimento de onda é 1,5406 ̊. Os ensaios foram realizados a temperatura de $298 \mathrm{~K}$ (ambiente), com tensão de $40 \mathrm{kV}$, corrente de $30 \mathrm{~mA}$, passo de $0,01^{\circ}$, tempo por passo de $0,6 \mathrm{~s}$ e o ângulo $2 \theta$ variando de $20 \mathrm{a} 80^{\circ}$.

\subsection{Elaboração dos compósitos alumínio/quasicristal}

Para a produção dos compósitos foram adicionados, em proporções volumétricas, $(0 \%, 2,5 \%, 5 \%$ e 10\%) do 
pó quasicristalino na matriz de alumínio. O alumínio foi fundido em um forno mufla laboratorial da marca Jung, modelo LF0912 no interior de um cadinho de carbeto de silício a temperatura de $750^{\circ} \mathrm{C}$. Em seguida, o cadinho foi retirado do forno e então, foram adicionadas as partículas quasicristalinas nas proporções em volume correspondentes para cada amostra. Realizou-se a mistura das partículas quasicristalinas no metal fundido manualmente com o auxílio de um tubo de quartzo, e posteriormente, essa mistura foi vertida em um molde de aço, dimensões apresentadas na Figura 1a, para solidificação do compósito.

O corte das amostras foi realizado em uma cortadora metalográfica da marca Teclago, modelo CM 80. A Figura 1 mostra o esquema dos cortes realizados nos compósitos. A Figura 1a, b, c e d representam, respectivamente, as seções antes do processo de laminação e as destinada à laminação, Na seção (SC) da Figura $1 \mathrm{~b}$, foram realizadas análises de MEV e microdureza Vickers dos compósitos obtidos diretamente do método stir casting, região de cor amarela com medidas de 3,5x3,5mm. Na Figura 1c, tem-se o esquema de corte realizado para obtenção da placa para o processo de laminação (região de cor laranja). A Figura 1d apresenta o corpo de prova destinado à laminação, onde na região (SL) de cor amarela com medidas de 2,0x3,5mm, foram realizadas análises de MEV e Microdureza Vickers após o processo de conformação do corpo de prova por laminação.

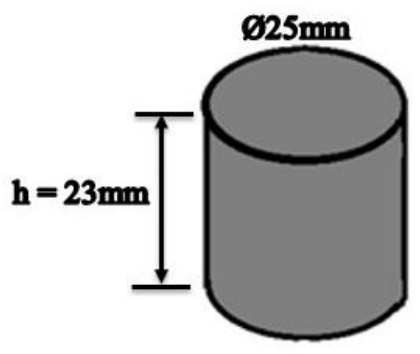

a)

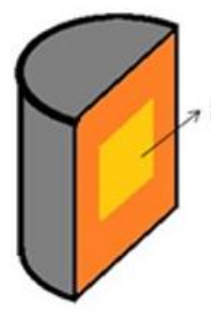

b)

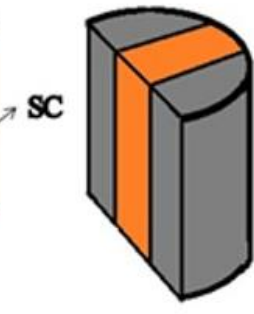

c)

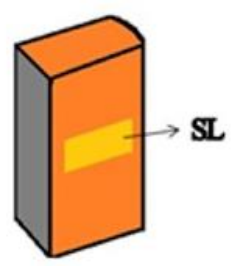

d)

Figura 1: Representação esquemática das seções dos compósitos alumínio/quasicristal antes e após processo de conformação por laminação.

\subsection{Laminação dos compósitos alumínio/quasicristal}

Para a laminação das placas retiradas dos compósitos, foi utilizado um laminador elétrico da marca Máquinas Coelhos e modelo LE200, com cilindros de 110 mm de diâmetro. A laminação das amostras ocorreu a temperatura ambiente, fixando a quantidade de 20 passes para cada amostra. Ao final de cada 4 passes, foi realizado um tratamento térmico de recuperação por $10 \mathrm{~min}$ à $150^{\circ} \mathrm{C}$ em um forno mufla laboratorial da marca Jung, modelo LF0912 e resfriamento a temperatura ambiente.

O percentual de redução à frio $(\mathrm{RF})$ de uma folha ou placa pode ser calculado através da Equação I a seguir [19]:

$$
\%(R F)=[(h i-h f) / h i] x 100
$$

Onde: hi e hf são, respectivamente, as espessuras iniciais e finais do corpo.

As amostras sofreram reduções percentuais de aproximadamente $40 \%$.

\subsection{Microscopia Eletrônica de Varredura}

As amostras foram caracterizadas utilizando um Microscópio Eletrônico de Varredura do tipo Leo 1430. Utilizou-se nas imagens o detector de elétrons secundários. As imagens foram obtidas utilizando-se uma distância focal (WD) entre 12 e 16mm, uma tensão entre 5 e $20 \mathrm{kV}$ e o spot size que variou entre 100 e 350.

\subsection{Microdureza Vickers}

Nesse ensaio usou-se um equipamento HMV Hardness Tester da marca Shimadzu com uma carga de 0,5N mantida por 15 segundos. Utilizou-se um penetrador de diamante de base quadrada e com ângulo de $136^{\circ}$ entre faces opostas. Foram realizadas 25 indentações na seção transversal do compósito direto da fundição 
(SC) e 20 indentações na superfície do laminado (SL).

\section{RESULTADOS E DISCUSSÕES}

\subsection{Difração de raios-X da liga quasicristalina AICuFeMn}

As ligas quasicristalinas revelaram a presença de duas fases mais evidenciadas, as quais foram identificadas através do banco de dados do software de análise de difração. São elas, uma fase quasicristalina - $i$ e uma fase cúbica do tipo $\mathrm{CsCl} \beta-\mathrm{Al}(\mathrm{Fe}, \mathrm{Cu})$.

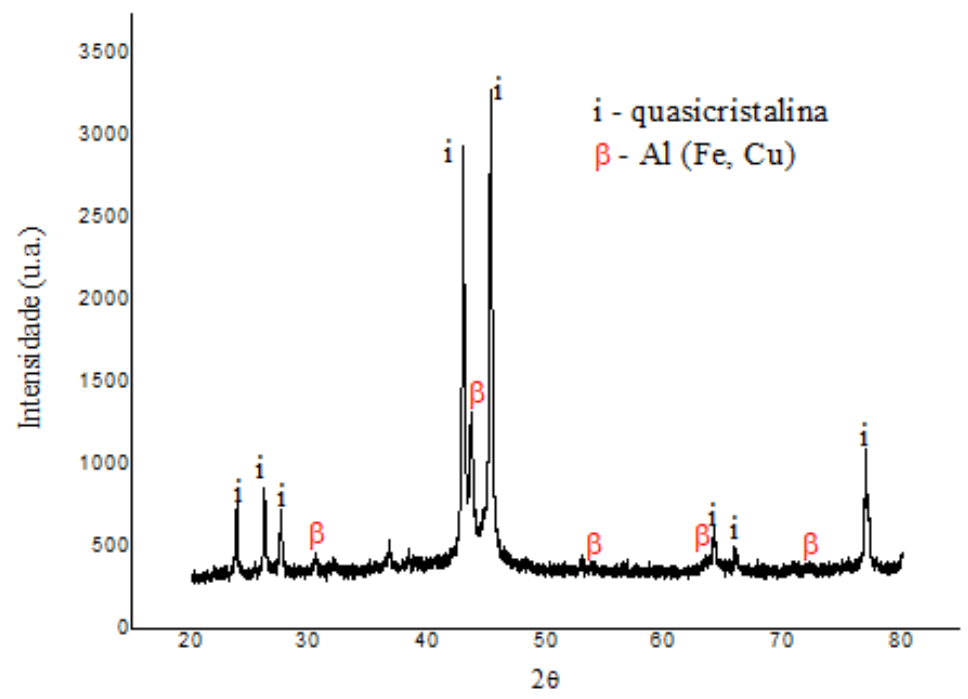

Figura 2: Difratograma da liga quasicristalina AlCuFeMn após tratamento térmico.

\subsection{Microscopia Eletrônica de Varredura dos compósitos alumínio/quasicristal}

A Figura 3 mostra as micrografias dos compósitos de matriz alumínio (Al) com reforço de partículas quasicristalinas (QC) obtidos diretamente do processo stir casting.

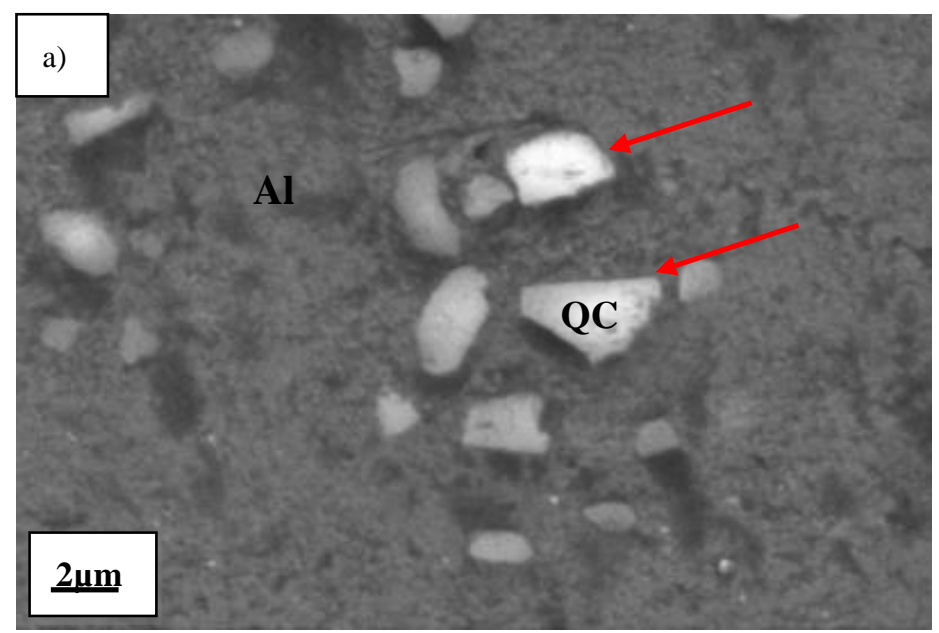



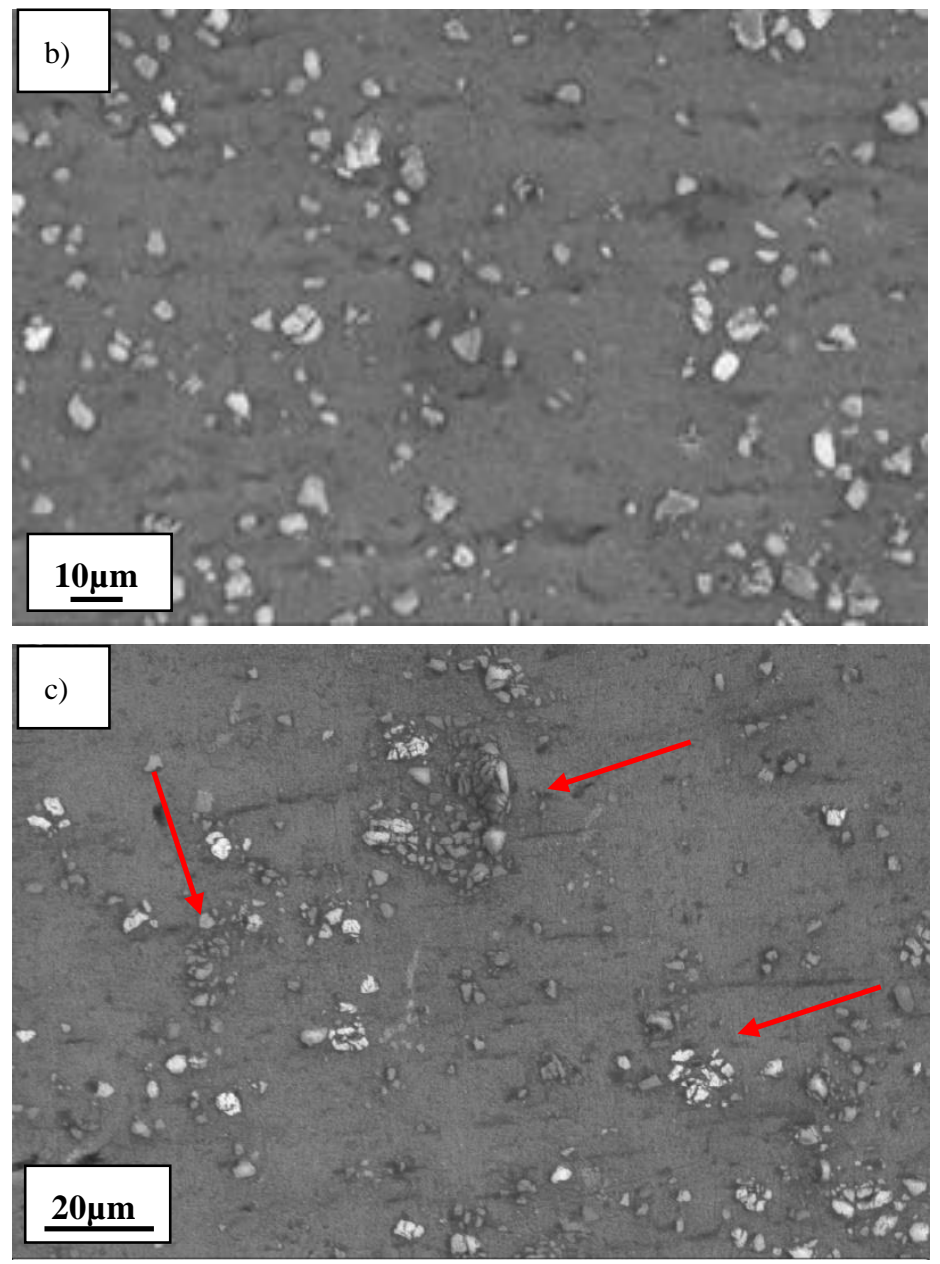

Figura 3: Micrografia dos compósitos obtidos diretamente do método stir casting. a) 2,5\% de QC; b)5\% de QC e c) $10 \%$ QC.

Verifica-se na Figura 3a que a matriz de alumínio circunda toda a partícula da liga quasicristalina. Em particular, por causa da natureza metálica da liga quasicristalina AlCuFeMn, uma boa compatibilidade de interface matriz/partícula foi observada, o que pode ser relacionado com um processo de interdifusão dos componentes do reforço com a matriz [1]. Mesmo com as baixas tensões superficiais dos quasicristais que promovem uma adesão mais fraca, é observada uma interface bem definida e um contato íntimo entre a matriz de alumínio e partícula quasicristalina (indicação por setas na Figura 3a).

A Figura 3 b mostra a distribuição de partículas do compósito de matriz de alumínio com 5\% em volume do reforço de partículas quasicristalinas. As partículas quasicristalinas (cor cinza claro) apresentam forma irregular, resultado do processo de moagem de alta energia [21]. É possível observar as partículas de QC dispersas de maneira relativamente uniforme na matriz de alumínio. Para uma maior fração volumétrica de reforço, Figura 3c, que mostra a micrografia do compósito com 10\% de partículas de QC, pode-se observar partículas aglomeradas em algumas áreas (indicação por setas na Figura 3c) [6].

\subsection{Microdureza Vickers}

A Figura 4 mostra a média das durezas calculadas e o desvio padrão (sd) para os compósitos obtidos diretamente do método stir casting e após passarem pelo processo de conformação a frio por laminação. 


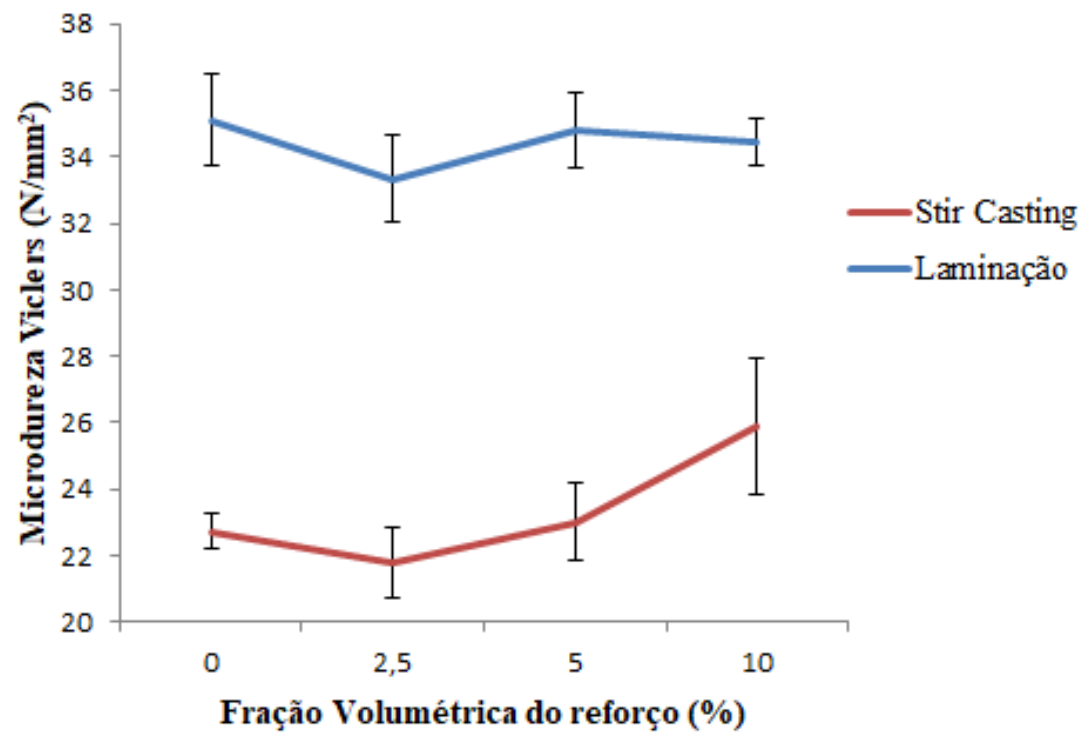

Figura 4: Microdureza Vickers dos compósitos alumínio/quasicristal obtidos diretamente do método stir casting e após o processo de conformação por laminação.

A força da matriz pode ser avaliada através de medidas de microdureza da matriz com reforço, desde que as indentações sejam pequenas e afastadas o suficiente para ficarem longe das partículas de reforço. A influência da fração volumétrica das partículas de QC na microdureza da matriz é mostrado claramente na Figura 6. Estes resultados revelaram uma dispersão acentuada, pois o comportamento da dureza em compósitos está intrinsecamente relacionado à distribuição do reforço na matriz, ou seja, a presença de aglomerados e de porosidades ocasionados pelo reforço pode influenciar diretamente na dureza final [19]. O aumento na dureza dos compósitos está intimamente relacionado ao aumento na fração volumétrica de reforço das partículas quasicristalinas [6].

Pôde-se observar um aumento na dureza dos compósitos conformados por laminação a frio em relação aos compósitos obtidos diretamente do método stir casting. Houve um aumento da dureza na média de $34,62 \%$ para os laminados com $0,2,5$ e $5 \%$ de reforço, e na média de $24,80 \%$ para o compósito com $10 \%$ de reforço na matriz de alumínio. Foi observado que os compósitos laminados apresentam valores de dureza mais elevados em todas as frações volumétricas de partículas quasicristalinas, o que pode ser explicado pelo estado de tensões residuais geradas no processo de conformação por laminação a frio. Assim como, o processo de laminação à frio ajuda a obter uma estrutura refinada e homogênea, removendo vazios e micro-vazios, e também ajuda a redistribuir as partículas de reforço, resultando em considerável eliminação de aglomerados de partículas e segregação. A eliminação de uma quantidade considerável de defeitos no compósito pelo processo de laminação a frio ajuda a aumentar a capacidade de endurecimento nesses materiais, o que pôde ser confirmado pelo aumento na dureza dos compósitos laminados em relação aos obtidos diretamente da fundição (stir casting) [22].

A tendência de aumento da dureza em relação ao aumento da fração do reforço quasicristalino $\mathrm{AlCu}-$ FeMn na matriz de alumínio, condiz com resultados obtidos por trabalhos disponíveis em periódicos, como em $[3,4,6]$, para compósitos alumínio/quasicristal obtido por outras rotas de processamento.

\section{CONCLUSÕES}

-Foi possível produzir compósitos com 2,5\%, 5\% e 10\% em volume da liga quasicristalina AlCuFeMn na matriz de alumínio com os métodos de processamento stir casting e conformação por laminação à frio.

-A microscopia eletrônica de varredura mostrou uma boa distribuição das partículas quasicristalinas na matriz de alumínio, mostrando que a matriz de alumínio circunda toda partícula quasicristalina. Com o aumento da fração volumétrica do reforço, pôde-se observar uma tendência em formação de aglomerados.

-Houve um aumento de aproximadamente $30 \%$ na dureza dos compósitos conformados por laminação a frio em relação aos compósitos obtidos diretamente do método stir casting. 


\section{AGRADECIMENTOS}

Apoio financeiro do Conselho Nacional de Desenvolvimento Científico Tecnológico (CNPq) e pelo apoio de materiais e equipamentos do Laboratório de Solidificação Rápida (LSR) da Universidade Federal da Paraíba (UFPB).

\section{BIBLIOGRAFIA}

[1] LAPLANCHE, G., JOULAIN, A., BONNEVILLE, J., et al., "Microstructures and mechanical properties of Al-base composite materials reinforced by $\mathrm{Al}-\mathrm{Cu}-\mathrm{Fe}$ particles", Journal of Alloys and Compounds, v. 493, pp. 453-460, Dec. 2009.

[2] SHAITURA, D. S., TEPLOV, A.A., CHIKINA, E.A., et al., "Composites reinforced by Al-Cu-Fe quasicrystalline particles with a copper matrix and their tribological properties", Crystallography Reports, v. 4, n. 6, pp. 951-955, Mar. 2010.

[3] KALOSHKIN, S. D., TCHERDYNTSEV, V. V., LAPTEV, A. I., et al., "Structure and mechanical properties of mechanically alloyed Al/Al-Cu-Fe composites", Journal of Materials Science, v. 39, pp. 5399-5402, Feb. 2004.

[4] PASSOS, T. A., "Estudo da viabilidade tecnológica de fabricação de compósitos alumínio-quasicristal por extrusão a quente", Tese de D.Sc., UFPB, João Pessoa, PB, Brasil. 2006.

[5] CHERDYNTSEV, V. V., KALOSHKIN, S. D., TOMILIN, I. A., et al., "Structure and properties of mechanically alloyed composite materials $\mathrm{Al} / \mathrm{Al}-\mathrm{Cu}-\mathrm{Fe}$ quasicrystal", The Physics of Metals and Metallography, v. 104, n. 5, pp. 497-504, Mar. 2007.

[6] LITYŃSKA-DOBRZYŃSKA, L., MITKA, M., GÓRAL, A., et al., "Microstructure and mechanical properties of aluminium matrix composites reinforced by $\mathrm{Al}_{62} \mathrm{Cu}_{25.5} \mathrm{Fe}_{12.5}$ melt spun ribbon", Materials Characterization, v. 117, pp. 127-133, May, 2016.

[7] PANWAR, N., CHAUHAN, A., "Fabrication methods of particulate reinforced aluminium metal matrix composite - A review", Materials Today: Proceedings, vol. 5, pp. 5933-5939, 2018.

[8] KANDPAL, B. C., KUMAR, J., SINGH, H., "Manufacturing and technological challenges in Stir casting of metal matrix composites - A Review", Materials Today: Proceedings, v. 5, pp. 5-10, Jul. 2016.

[9] SARAVANAN, C., SUBRAMANIAN, K., SIVAKUMAR, D. B., et al., "Fabrication of aluminium metal matrix composite -a review", Journal of Chemical and Pharmaceutical Sciences, v. 7, pp. 82-87, Mar. 2015.

[10] ANNIGERI, U. K., KUMAR, G. V. B., "Method of stir casting of aluminum metal matrix composites: A review", Materials Today: Proceedings, v. 4, pp. 1140-1146, 2017.

[11] HELMAN, H., CETLIN, P.R., Fundamentos da conformação mecânica dos metais, Rio de Janeiro: Guanabara Dois, 1983.

[12] ZHANG, C., LIU, S., ZHANG, C., "Micro crack of aluminum sheet during cold rolling", World Journal of Mechanics, v. 1, pp. 169 - 175, Aug. 2011.

[13] GOMES, M. A., Caracterização mecânica e microestrutural de compósitos de matriz metálica $\mathrm{Al} / \mathrm{SiC}_{\mathrm{p}} \mathrm{e}$ $\mathrm{Al} / \mathrm{Al}_{2} \mathrm{O}_{3 \mathrm{p}}$ obtidos via interação por laminação Acumulativa, Dissertação de M.Sc., USP, Escola de Engenharia de São Carlos, São Carlos, SP, Brasil. 2015.

[14] HUTTUNEN-SAARIVIRTA, E., VUORINEN, J., "Preparation and characterization of melt-spun Al$\mathrm{Cu}-\mathrm{Fe}$ quasicrystals", Intermetallics, v. 13, pp. 885-895, Feb. 2005.

[15] GUO, X., CHEN, J., YU, H., et al., "A study on the microstructure and tribological behavior of coldsprayed metal matrix composites reinforced by particulate quasicrystal", Surface \& Coatings Technology, v. 268, pp. 94-98, Jun. 2015.

[16] LITYŃSKA-DOBRZYŃSKA, L., DUTKIEWICZ, J., STAN-GLOWINSKA, K., et al., "Characterization of aluminium matrix composites reinforced by $\mathrm{Al}-\mathrm{Cu}-\mathrm{Fe}$ quasicrystalline particles", Journal of Alloys and Compounds, v. 643, pp. 114-118, Sep. 2015.

[17] HUTTUNEN-SAARIVIRTA, E. H., "Microstructure, fabrication and properties of quasicrstalline AlCu-Fe Alloys: a review", Journal of Alloys Compounds, v. 363, pp. 150-174, Mar. 2004.

[18] YOKOYAMA, Y., FUKAURA, K., SUNADA, H., et al., "Production of single $\mathrm{Al}_{64} \mathrm{Cu}_{23} \mathrm{Fe}_{13}$ icosahedral quasicrystal with the Czochralski method", Materials Science and Engineering, v. 294, pp. 68-73, Dec. 2000 
[19] FLEURY, E, LEE, S. M, CHOI, G., et al., "Comparison of Al-Cu-Fe quasicrystalline particle reinforced Al composites fabricated by conventional casting and extrusion”, Journal of Materials Science, v. 36, pp. 963-970, 2001.

[20] SMITH, W. F., HASHEMI, J., Fundamentos de engenharia e ciência dos materiais, 5.ed, Porto Alegre, AMGH, 2012.

[21] CHIAVERINI, V., Metalurgia do pó, 4 ed., São Paulo, Associação Brasileira de Metalurgia e Materiais, 2001.

[22] ALANEME, K. K., "Mechanical behavior of cold deformed and solution heat-treated alumina reinforced AA 6063 metal matrix composites", The West Indian Journal of Engineering, v. 35, n. .2, pp.31-35, Jan. 2013.

\section{ORCID}

Maria Aline Martins Gonzaga Tibério Andrade dos Passos

Rodinei Medeiros Gomes https://orcid.org/0000-0002-0514-8755

https://orcid.org/0000-0002-1333-286X

https://orcid.org/0000-0002-4317-4567 